\title{
Déterminer la pondération de la consommation des ressources en chimiothérapie ambulatoire liée à la charge de travail des infirmières
}

par Esther Green, Colin Preyra, Janice Stewart, Cindy McLennan, Rosemary Bland, Tamara Dus, Marcia Langhorn, Kathy Beattie, Annie Cheung, Sherrie Hertz, Haim Sechter, Judy Burns, Helen Angus, Carol Sawka

\section{Abrégé}

Les programmes de cancérologie ontariens se sont fixés comme objectif de dispenser des soins infirmiers de haute qualité et des traitements sécuritaires à la fois pour les patients et pour le personnel. Dans le domaine de la santé, les contraintes financières inhérentes à la prestation des soins exigent que les mécanismes de financement rendent compte non seulement du coût des médicaments mais encore de facteurs tels que les coûts des ressources humaines en pharmacie et en soins infirmiers. Alors que certaines organisations ont élaboré des systèmes de classification des patients afin de mesurer l'intensité des soins infirmiers et la charge de travail des infirmières, ces systèmes s'appliquent principalement aux clientèles hospitalisées et présentent de nombreux défis tels que la nécessité pour les infirmières de documenter la charge de travail exigée par les soins à des fins de justification. Cet article a pour but de tracer les grandes lignes de la méthodologie et de l'implication des infirmières en vue d'élaborer une pondération de la consommation des ressources basée sur les protocoles pouvant être appliquée aux salles de chimiothérapie ambulatoire. La méthodologie inclut la détermination de la charge de travail reliée au temps dont les infirmières ont besoin pour préparer, conseiller et évaluer les patients et leur fournir l'enseignement requis ainsi que le temps nécessaire pour rassembler les fournitures, accéder aux lignes de perfusion, surveiller, gérer les effets indésirables, gérer les symptômes et documenter les soins. La pondération de la consommation des ressources fournit une meilleure mesure de la complexité des soins exigés par les patients atteints de cancer traités en milieu ambulatoire.

\section{Au sujet des auteurs}

Esther Green, inf., B.Sc.inf., M.Sc.(Ens.), Action Cancer Ontario

Colin Preyra, Ph.D., Institut de recherche en services de santé, Université de Toronto

Janice Stewart, inf., B.Sc.inf., CSIO(C), Hôpital Princess Margaret, Réseau universitaire de santé

Cindy McLennan, inf., MBA, CSIO $(C)$, Centre de cancérologie de L'Hôpital d'Ottawa

Rosemary Bland, inf., B.Sc.inf., $\operatorname{CSIO}(C)$, ICSP(C), Centre de cancérologie Juravinski, Hamilton Health Sciences

Tamara Dus, inf., M.Sc.inf., CSIO(C), Centre régional de cancérologie R.S. McLaughlin de Durham

Marcia Langhorn, inf., CSIO(C), Centre régional de cancérologie, Centre des sciences de la santé de London

Kathy Beattie, inf., CSIO(C), Centre de cancérologie Odette, Centre des sciences de la santé Sunnybrook

Annie Cheung, R.Ph., B.Sc.(Pharm.), Action Cancer Ontario

Sherrie Hertz, B.Sc.(Pharm.), Action Cancer Ontario

Haim Sechter, M.B.A., Action Cancer Ontario

Judy Burns, B.Sc.inf., M.Sc.S., CHE, Action Cancer Ontario

Helen Angus, B.A.(Hons), M.Sc.Plan., Action Cancer Ontario

Carol Sawka, MD, FRCPC, Action Cancer Ontario

\section{Introduction}

Le système de lutte contre le cancer de l'Ontario, Canada, met l'accent sur la qualité, l'innovation et la gestion du rendement. Action Cancer Ontario (ACO) est une organisation guidée par les données; elle donne des conseils au gouvernement provincial en se basant sur les preuves disponibles et met à profit les meilleures données probantes afin d'éclairer les décisions en matière de financement, à la fois pour les traitements et pour les dépenses en immobilisations.

Comme le système de lutte contre le cancer bénéficie d'un financement public, ses gestionnaires s'efforcent de trouver des méthodes donnant une mesure exacte des coûts associés à la dispensation des soins aux patients traités en ambulatoire. En 1999, le Groupe de travail sur la thérapie générale d'ACO, composé d'infirmières, de pharmaciens et d'oncologues médicaux, a examiné la charge de travail liée aux ressources humaines en ce qui a trait à la thérapie générale. Le groupe a utilisé les données et les résultats de recherche disponibles afin de parvenir à un consensus relativement à la détermination de la charge de travail du personnel infirmier et de celle du personnel de pharmacie. Aucun outil d'analyse méthodologique n'étant disponible à l'époque, le groupe a donné son aval à l'emploi d'une mesure rudimentaire: 1700 visites de chimiothérapie par ETP d'infirmière, selon les recommandations de la BC Cancer Agency (Systematic Therapy Taskforce, 2000). La mesure ne tenait pas compte des types de protocoles de chimiothérapie, de la complexité des soins ni de la nature de la clientèle. En 2002, la charge

\section{Définition de termes}

Thérapie générale pour le cancer: médicaments de chimiothérapie et liés aux soins de soutien administrés par voie intraveineuse, par voie intrapéritonéale ou par voie intrathécale. On utilise également le terme "traitement systémique"

Pondération de la consommation des ressources: la mesure moyenne des ressources exigée par la prestation des services de thérapie générale, déterminée par les ressources réellement utilisées (comme le temps passé au fauteuil de chimiothérapie, les ressources relatives aux soins infirmiers et de pharmacie) et liées à la clientèle concernée

Oncologie ambulatoire: soins de cancérologie fournis en clinique externe

Salle de chimiothérapie: milieu spécialisé au sein d'une unité de soins ambulatoires où les infirmières administrent la thérapie générale

Besoins en matière de ressources humaines: personnel exigé pour la prestation des soins et traitements anticancéreux

Temps lié aux soins infirmiers directs: temps de soins infirmiers nécessaires à l'évaluation et à la prise en charge des patients dans le point de prestation de soins

Temps lié aux soins infirmiers indirects: le temps consacré à la préparation de l'administration du traitement et le temps posttraitement

Protocole: groupe de médicaments de chimiothérapie/de soutien qui sont administrés ensemble selon un horaire particulier pour un type de cancer donné

Enseignement aux patients: l'enseignement dispensé aux patients et à leur famille à propos de la maladie, du traitement, des effets secondaires éventuels et de la gestion des symptômes

Indice des groupes clients (IGC): Un système canadien de classification des patients élaboré par l'Institut canadien d'information sur la santé et basé sur le diagnostic principal qui sert à regrouper les patients ayant reçu leur congé des hôpitaux de soins actifs 
de travail a été révisée à la baisse à 1500 visites de chimiothérapie par ETP d'infirmière (Systematic Therapy Advisory Working Group [STAWG], 2002) lorsqu'il a été reconnu que le chiffre initial n'était pas réaliste du fait des changements liés aux nouveaux protocoles de traitement; mais une fois de plus, il n'était pas tenu compte de la complexité des protocoles ni de celle des soins requis par la clientèle comme le type de cancer, l'âge ou la présence d'une comorbidité.

La recherche documentaire a révélé que peu d'outils avaient été développés dans le but de mesurer l'intensité de l'utilisation des ressources infirmières en lien avec la thérapie générale anticancéreuse. Seul un article de Cusack, Jones et Chisholm (2004a), examiné plus loin dans cet article, décrivait le développement d'un outil de mesure de consommation des ressources en oncologie. En 2006, le comité du Programme de soins infirmiers oncologiques (ONPC) d'ACO a lancé un dialogue ciblé entre les chefs de file et les gestionnaires des soins infirmiers, les infirmières de chevet et les infirmières en pratique avancée en vue de se pencher sur le développement éventuel d'un outil permettant de définir la complexité des soins associés aux protocoles de chimiothérapie utilisés dans les programmes de cancérologie de tout l'Ontario. Cela faisait déjà quelque temps que les membres de l'ONPC avaient fait état des limitations liées au consensus sur la charge de travail établissant cette dernière à 1500 visites de chimiothérapie par infirmière (STAWG, 2002); beaucoup de gestionnaires s'inquiétaient de ne pas pouvoir décrire les situations où un nombre moindre de patients étaient traités par le même nombre d'infirmières, c'est-à-dire que des infirmières effectuaient moins de 1500 visites. Les gestionnaires avaient l'intuition que les patients exigeaient des soins infirmiers de plus grande intensité dans le cadre de leur traitement, mais il n'existait pas de données leur permettant de déterminer la complexité de ces soins ou d'expliquer l'accroissement des heures ou des coûts de soins infirmiers.

En 2007, ACO a rassemblé un groupe d'experts composé de cadres infirmières qui étaient gestionnaires ou administratrices (M.Sc., MBA, M.Sc.inf.), des pharmaciens en oncologie (B.Pharm.), une économiste de la santé (Ph.D.) émanant du milieu universitaire et des infirmières de chevet (CSIO(C), inf.) ayant une excellente connaissance de la thérapie générale, afin de mettre au point la méthodologie qui servirait à déterminer la pondération de la consommation des ressources associée à 177 protocoles de chimiothérapie distincts lesquels constituaient un total de 297 protocoles applicables à des sièges particuliers du cancer.

Cet article contient divers termes qui pourraient ne pas être bien connus de l'ensemble des lecteurs. Les auteurs ont ainsi défini chacun de ces termes à des fins de clarté (voir la case Définitions de termes).

\section{Revue de la littérature}

Le groupe d'experts a d'abord effectué une revue de la littérature en recherchant les conseils d'infirmières ayant étudié les enjeux concernant la charge de travail des infirmières. Cette revue de la littérature a révélé d'intéressantes variations en matière de développement et de mise en œuvre d'outils (voir le tableau 1). Par exemple, de nombreux concepts et outils ont été élaborés pour quantifier et définir le travail infirmier et ce, dans le but de résoudre des problèmes tels que l'équilibre de la charge de travail des infirmières, le plus souvent en ce qui a trait aux soins aux patients hospitalisés. Des outils comme l'affectation de patients aux infirmières (Welton, Zone-Smith \& Bandyopadhyay, 2009), le système de mesure du volume des soins ambulatoires (Cusack, Jones \& Chisholm, 2004a, 2004b; Jones, Cusack \& Chisholm, 2004; Moore \& Hastings, 2006), des systèmes de classification des patients (Chabot \& Fox, 2005; Harper \& McCully, 2007) ainsi que des systèmes d'ordonnancement des soins (Langhorn \& Morrison, 2001a; 2001b) sont des types de systèmes de mesure qui permettent de planifier les ressources infirmières en contexte ambulatoire. Certains des outils faisaient appel à des études des temps et mouvements ou se basaient sur la quantification rétrospective objective du temps passé auprès des patients tandis que d'autres combinaient les deux méthodes pour développer les outils (Cusack et al., 2004a, 2004b; Colombo, Solberg, Vanderhoeft, Ramsay \& Schouten, 2005).

Les études des temps et mouvements ont pour but de mesurer objectivement l'affectation des ressources infirmières. Comme ces outils se concentrent sur les tâches et sur les éléments physiques des soins, ils ignorent les aspects sociaux ou psychologiques des soins infirmiers ainsi que les éléments évaluation initiale, planification, intervention et évaluation du champ d'exercice infirmier (Harper \& McCully, 2007). Huber et Craig (2007) reconnaissent que les études des

\begin{tabular}{|c|c|c|c|}
\hline Auteurs et année & Outil & Type & But \\
\hline Chabot \& Fox (2005) & $\begin{array}{l}\text { Système de } \\
\text { classification des } \\
\text { patients }\end{array}$ & Consensus infirmier & $\begin{array}{l}\text { Mesure de la charge de travail relativement à } \\
\text { l'ordonnancement de la chimiothérapie }\end{array}$ \\
\hline $\begin{array}{l}\text { Harper \& McCully } \\
(2007)\end{array}$ & $\begin{array}{l}\text { Système de } \\
\text { classification des } \\
\text { patients; outils sur la } \\
\text { lourdeur des tâches }\end{array}$ & $\begin{array}{l}\text { Études des temps et mouvements } \\
\text { ou liste de contrôle des soins aux } \\
\text { patients où des indices de lourdeur } \\
\text { des tâches ont été attribués }\end{array}$ & $\begin{array}{l}\text { Pour prévoir les besoins de dotation et contrôler plus } \\
\text { étroitement les ratios infirmière/usagers. }\end{array}$ \\
\hline $\begin{array}{l}\text { Colombo, Solberg, } \\
\text { Vanderhoeft, Ramsay } \\
\& \text { Schouten (2005) }\end{array}$ & $\begin{array}{l}\text { Nom de l'outil non } \\
\text { fourni }\end{array}$ & $\begin{array}{l}\text { Combinaison d'une étude des } \\
\text { temps et mouvements et d'une } \\
\text { étude rétrospective }\end{array}$ & $\begin{array}{l}\text { Planification des niveaux de dotation infirmière et } \\
\text { évaluation de la charge de travail dans les services } \\
\text { d'hématologie-oncologie }\end{array}$ \\
\hline $\begin{array}{l}\text { Cusack, Jones \& } \\
\text { Chisholm (2004); } \\
\text { Moore \& Hastings } \\
\text { (2006) }\end{array}$ & $\begin{array}{l}\text { Système d'intensité } \\
\text { des soins } \\
\text { ambulatoires }\end{array}$ & $\begin{array}{l}\text { Étude rétrospective des } \\
\text { déclarations des infirmières }\end{array}$ & $\begin{array}{l}\text { Planification des niveaux de dotation infirmière et } \\
\text { évaluation de la charge de travail dans des contextes } \\
\text { d'hôpital de jour: chimiothérapie ambulatoire, } \\
\text { médecine/chirurgie, pédiatrie et hématologie/oncologie }\end{array}$ \\
\hline $\begin{array}{l}\text { Langhorn \& Morrison } \\
(2001)\end{array}$ & $\begin{array}{l}\text { Système } \\
\text { d'ordonnancement } \\
\text { de la chimiothérapie }\end{array}$ & $\begin{array}{l}\text { Unités pondérées de complexité } \\
\text { Opis (intensité/procédure } \\
\text { normalisée des soins infirmiers } \\
\text { en minutes) et «Nursing Workload } \\
\text { System» de l'Alberta Cancer Board }\end{array}$ & $\begin{array}{l}\text { Mesure de la charge de travail relativement à } \\
\text { l'ordonnancement de la chimiothérapie }\end{array}$ \\
\hline $\begin{array}{l}\text { Welton, Zone-Smith } \\
\text { \& Bandyopadhyay } \\
\text { (2009) }\end{array}$ & $\begin{array}{l}\text { Dossier d'affectation } \\
\text { de patients aux } \\
\text { infirmières }\end{array}$ & $\begin{array}{l}\text { Étude rétrospective des } \\
\text { déclarations des infirmières et } \\
\text { base de données d'intensité des } \\
\text { soins infirmiers }\end{array}$ & $\begin{array}{l}\text { Calculer l'intensité des soins infirmiers et les coûts } \\
\text { des soins infirmiers directs }\end{array}$ \\
\hline
\end{tabular}


temps et mouvements ne tiennent aucunement compte des aspects complexes et multidimensionnels des soins mentionnés par Harper et McCully (2007). En outre, les interventions infirmières peuvent être réalisées simultanément, individuellement ou consécutivement (Huber \& Craig, 2007). La diversité des interventions sélectionnées par les infirmières et les variations de l'horaire choisi pour les dispenser contribuent également à la complexité de la mesure des soins. Les études des temps et mouvements visent à mesurer ce qui se produit réellement plutôt que ce qui se produirait en vertu d'une norme de soins de référence. De plus, l'élaboration de ces études est coûteuse et nécessite beaucoup de temps, et les méthodes d'échantillonnage du travail peuvent souffrir de l'effet Hawthorne où les sujets changent leurs habitudes de travail du seul fait d'être observés (Colombo et al., 2005). Finalement, l'ordonnancement ou la détermination des coûts liés aux soins infirmiers constituaient habituellement les prémisses à l'origine du développement de ces outils.

Le concept de catégories définies «d'intensité» ou de niveaux de soins, liées à la quantité de temps exigée pour l'administration de chaque protocole et la notion de niveaux de gravité sont des éléments essentiels qui doivent être pris en compte dans les outils pertinents (Chabot \& Fox, 2005; Langhorn \& Morrison, 2001a; 2001b). Unruh (2008) soulignait la nécessité de disposer d'une mesure de la charge de travail basée sur des données probantes et normalisée permettant d'évaluer tous les effets des facteurs contributifs connus y compris le temps infirmier direct et indirect (Morris, MacNeela, Scott, Treacy \& Hyde, 2007). Quoique divers outils aient été élaborés en vue de mesurer la gravité de l'état des patients et/ou l'intensité des soins infirmiers, chaque outil a des avantages et des inconvénients. Il existe peu d'écrits appuyant l'affectation des ressources infirmières à un microniveau (p. ex. une clinique de chimiothérapie ambulatoire) et encore moins qui appuieraient la prise de décision à un macroniveau (p. ex. au niveau provincial ou régional). Dans les milieux ambulatoires d'administration des traitements anticancéreux systémiques, les besoins du patient en matière de ressources sont étroitement reliés à son protocole de traitement, et pourtant, les classifications disponibles ne reconnaissent pas cette variation. Le présent article a pour but de tracer les grandes lignes du développement d'une pondération de la consommation des ressources basée sur les protocoles et applicable aux salles de chimiothérapie ambulatoire.

\section{Méthodologie \\ Le groupe d'experts}

Un groupe d'experts a été formé en réunissant une économiste de la santé (Ph.D.) du milieu universitaire, des pharmaciens (B.Pharm.) ayant une expertise en oncologie, des cadres infirmières (M.Sc., M.Sc. inf., CSIO(C)), des infirmières en oncologie (inf., CSIO(C)) et des gestionnaires (MBA) ayant une excellente connaissance de la thérapie générale. Cette initiative avaient deux buts principaux: 1) déterminer les soins infirmiers exigés pour prendre en charge les patients recevant des protocoles de chimiothérapie complexes, notamment les polychimiothérapies, pour tous les sièges de cancer, y compris les éléments des soins directs et indirects; et, 2) attribuer une pondération qui serait utilisée pour le financement de la thérapie générale dans les centres de cancérologie ontariens. Les membres du groupe d'experts ont été sélectionnés en fonction de leur expertise et de leur capacité à travailler en collaboration et à atteindre le consensus en privilégiant le dialogue. Plutôt que d'employer la méthodologie axée sur l'étude des temps et mouvements, et fort du savoir collectif de ses membres en matière d'implantation des protocoles de chimiothérapie dans la pratique, le groupe a utilisé une approche axée sur le consensus pour chacune des dimensions des soins infirmiers. Les infirmières figurant dans le groupe représentaient des programmes de cancérologie situés dans des établissements universitaires et dans des collectivités de taille moyenne. L'expérience des infirmières dans le domaine de l'oncologie variait de 10 à 20 ans. L'économiste et les autres membres du groupe possédaient une expérience pertinente de leurs domaines respectifs dont l'étendue variait de 5 à 10 ans.

\section{Le processus}

Les membres du groupe avaient une expertise des disciplines en jeu, une expérience des soins directs et de la gestion financière et enfin, des connaissances sur les médicaments utilisés pour traiter les différents types de cancers. Ce mélange d'expertise et de savoir a favorisé les discussions et les débats relatifs à chacun des éléments de la charge de travail, des définitions et des affectations. L'approche axée sur le consensus a été retenue pour plusieurs raisons importantes:

- L'accent pouvait être mis sur les affectations de la charge de travail en fonction des pratiques exemplaires par opposition aux pratiques actuelles;

- L'utilisation du consensus a sensiblement diminué le coût de l'étude;

- Les comparaisons avec les systèmes hospitaliers relatifs à la charge de travail, lesquels ont été calibrés à partir d'études des temps et mouvements, faisaient ressortir un haut degré de concordance avec les résultats de la présente étude;

- Le processus axé sur le consensus permettait d'examiner l'ensemble des protocoles;

- Les modèles et le cadre fondé sur le consensus élaborés dans le cadre de ce processus, peuvent être facilement mis à jour à mesure qu'apparaissent de nouveaux protocoles dans la pratique (les valeurs de pondération sont révisées chaque année au fur et à mesure de l'ajout de nouveaux protocoles);

- Enfin, la méthodologie axée sur le consensus n'exige pas du personnel infirmier qu'il saisisse de quelconques données, ce qui évite une charge de travail supplémentaire aux prestataires de soins directs.

$\mathrm{Au}$ début du processus, l'infirmière responsable (EG) a communiqué avec plusieurs établissements de soins afin de recueillir des renseignements sur leur emploi de mesures de la charge de travail dans les salles de chimiothérapie du Canada. La majorité des établissements contactés se servaient de versions modifiées de systèmes de classification des patients hospitalisés ou d'outils sur la gravité de leurs besoins; par contre, ils n'employaient pas de méthodes de saisie de la charge de travail en chimiothérapie à l'exception du nombre de patients traités, du volume d'utilisation des protocoles et du nombre d'infirmières œuvrant dans les salles de chimiothérapie.

La première phase du projet consistait à cerner toutes les activités associées à la charge de travail des infirmières. On s'est entendu sur les définitions pour toutes les activités et celles-ci ont été documentées afin d'appuyer les phases ultérieures. L'objectif était d'assurer l'intégralité du processus et d'établir une base de référence pouvant incorporer les variations d'un protocole à l'autre. Dès le lancement des travaux, on a convenu de plusieurs critères d'exclusion: les protocoles liés aux essais cliniques, les médicaments administrés par voie orale et les protocoles concernant les patients hospitalisés. Les protocoles liés aux essais cliniques ont été exclus parce que ces médicaments n'étaient ni financés ni approuvés à des fins de financement et n'étaient donc pas pertinents. Les médicaments administrés par voie orale ont été exclus parce qu'ils exigent une faible quantité de temps de la part du personnel infirmier. Quant aux protocoles concernant les patients hospitalisés, ils ont été exclus parce qu'ils représentaient une minorité des traitements effectués.

La deuxième phase des travaux a été réalisée de manière autonome par chacun des membres de l'équipe infirmière. Chaque membre du groupe d'experts déterminait, à partir de sa propre expérience, le temps requis pour chaque visite associée à un protocole particulier. Ensuite, le groupe examinait toute divergence au niveau des attributions de temps et cherchait à atteindre le consensus pour chaque protocole; le résultat ainsi décidé devenait l'attribution de temps finale pour chacune des activités exigées. Bien que dans certains cas, les valeurs de temps signalées à l'origine variaient d'un hôpital à l'autre, le groupe d'experts a veillé à ce que les valeurs de temps indiquées soient le reflet de soins de qualité. Il était manifeste que les autres activités en soins infirmiers variaient d'un protocole à un autre. On a 


\begin{tabular}{|c|c|c|c|}
\hline \multirow{2}{*}{$\begin{array}{l}\text { Colonne } \\
\text { A: }\end{array}$} & \multirow{2}{*}{\begin{tabular}{|l}
$\begin{array}{l}\text { Élément de la } \\
\text { charge de travail }\end{array}$ \\
Accès aux CVC / démarrage \\
et débranchement d'IV-20 \\
minutes pour chaque type \\
d'accès (p. ex. IV, IP)
\end{tabular}} & \multicolumn{2}{|c|}{ Définitions de chaque élément de la charge de travail associée à un protocole } \\
\hline & & $\begin{array}{l}\text { - Accéder aux cathéters centraux, rinçage et } \\
\text { évaluation de la perméabilité des CVC } \\
\text { - Prélèvement de sang au niveau du CVC, au } \\
\text { besoin } \\
\text { - Panser le site d'insertion du cathéter central }\end{array}$ & $\begin{array}{l}\text { - Accès à l'IV périphérique et évaluation de } \\
\text { cette dernière } \\
\text { - Débranchement et rinçage du CVC/de l'IV } \\
\text { - Ne s'applique pas à la sonde de gastrostomie } \\
\text { ou à l'accès intravésiculaire }\end{array}$ \\
\hline B: & $\begin{array}{l}\text { Temps de préparation } \\
15 \text { minutes }\end{array}$ & $\begin{array}{l}\text { - Examen des ordonnances de chimiothérapie, } \\
\text { analyses sanguines, allergies, évaluation } \\
\text { infirmière actuelle si le patient consulte la } \\
\text { clinique ou examen de l'autoévaluation si } \\
\text { dirigé directement vers la chimio } \\
\text { - Examen du dernier traitement } \\
\text { - Nouveau patient: examen des antécédents et } \\
\text { du rapport de consultation } \\
\text { - Examen des directives liées au protocole et à } \\
\text { son administration }\end{array}$ & $\begin{array}{l}\text { - Identification et rassemblement du matériel } \\
\text { nécessaire } \\
\text { - Préparation des lignes de rinçage } \\
\text { - Revêtir l'équipement de protection individuel } \\
\text { - S'assurer que le formulaire de consentement a } \\
\text { été signé }\end{array}$ \\
\hline C: & $\begin{array}{l}\text { Éducation } \\
15 \text { minutes }\end{array}$ & - Nouveau patient: éducation liée à sa chimio & $\begin{array}{l}\text { - Renforcement continuel de l'éducation à la } \\
\text { chimio à chaque visite du patient }\end{array}$ \\
\hline D: & $\begin{array}{l}\text { Documentation } \\
15 \text { minutes }\end{array}$ & $\begin{array}{l}\text { - Documentation (dossier en version papier ou } \\
\text { électronique): } \\
\text { - Accès aux IV/CVC } \\
\text { - Médicaments administrés } \\
\text { - Apparition de symptômes et toxicités } \\
\text { - Interventions requises }\end{array}$ & $\begin{array}{l}\text { - Documentation (dossier en version papier ou } \\
\text { électronique): } \\
\text { - réponse au traitement } \\
\text { - enseignement sur la chimio } \\
\text { - état lors du congé } \\
\text { - appels téléphoniques avec médecins, autres } \\
\text { professionnels de la santé, centre d'accès } \\
\text { aux soins communautaires }\end{array}$ \\
\hline E: & $\begin{array}{l}\text { Évaluation de la toxicité et } \\
\text { gestion des symptômes } \\
15 \text { minutes }\end{array}$ & $\begin{array}{l}\text { - Évaluation du patient s'il a été dirigé } \\
\text { directement vers la chimio } \\
\text { - Gestion des symptômes (utiliser des directives } \\
\text { médicales, le cas échéant) } \\
\text { - Graduation de l’INCC, au besoin }\end{array}$ & $\begin{array}{l}\text { - Interventions (p. ex. appeler le médecin, } \\
\text { prélèvements de sang, admissions éventuelles } \\
\text { à l’hôpital) }\end{array}$ \\
\hline F: & $\begin{array}{l}\text { Antiémétiques, } \\
\text { prémédications ou } \\
\text { médicaments additionnels } \\
5 \text { minutes pour chacun } \\
\text { des prémédicaments } \\
\text { antiémétiques simples ( } \leq 2 \\
\text { méd.) } \\
7 \text { minutes pour chacun } \\
\text { des prémédicaments } \\
\text { antiémétiques plus } \\
\text { complexes (3 méd. ou plus) }\end{array}$ & $\begin{array}{l}\text { - Administration des antiémétiques prescrits } \\
\text { - Obtenir l'ordonnance si le patient a oublié } \\
\text { de conserver l'antiémétique ou de le prendre } \\
\text { avant le traitement } \\
\text { - Administrer les prémédicaments par le biais } \\
\text { de la sonde de gastrostomie }\end{array}$ & $\begin{array}{l}\text { - Exiger une ordonnance si l'apparition d'un } \\
\text { symptôme est nouvelle ou non maîtrisée } \\
\text { - Administration d'un diurétique } \\
\text { - Obtenir les liquides dont le patient a besoin } \\
\text { pour prendre ses pilules }\end{array}$ \\
\hline G: & $\begin{array}{l}\text { Fourniture du temps des } \\
\text { infirmières lié au protocole }\end{array}$ & $\begin{array}{l}\text { - Vésicant gros volume=bolus de } 20 \text { minutes } \\
\text { (> } 50 \mathrm{ml}+\text { ) } \\
\text { - Vésicant petit volume=bolus de } 10 \text { minutes } \\
\text { - Autres bolus = } 5 \text { à } 10 \text { minutes } \\
\text { - Suspension de sac pour IV titré) =15 } \\
\text { minutes (paclitaxel, docetaxel) -N'inclut pas } \\
\text { l'intervention et l'observation directes } \\
\text { - Anticorps monoclonaux (rituximab) - } 30 \\
\text { minutes pour l'induction; } 15 \text { minutes pour la } \\
\text { maintenance } \\
\text { - Anticorps monoclonaux (trastuzumab, } \\
\text { cetuximab)=15 minutes } \\
\text { - Anticorps monoclonaux (panitumumab, } \\
\text { bevacizumab)=10 minutes } \\
\text { - Suspension de sac pour IV ordinaire =5 } \\
\text { minutes }\end{array}$ & $\begin{array}{l}\text { - Pompes (programmables)= } 30 \text { minutes } \\
\text { + } 15 \text { minutes pour la double vérification } \\
\text { indépendante-Total=45 minutes } \\
\text { - Pompes (Infusor/Intermate)= } 15 \text { minutes } \\
\text { (double signature infirmière, vérification du } \\
\text { patient, débranchement par le centre d'accès } \\
\text { aux soins communautaires) } \\
\text { - Ponction lombaire/intrathécale / réservoir } \\
\text { d'Ommaya (assistance infirmière) = } 30 \text { minutes } \\
\text { - Réservoir d'Ommaya (réalisé par } \\
\text { l'infirmière)=30 minutes } \\
\text { - Voie intrapéritonéale (paclitaxel/carboplatine } \\
\text { ou cisplatine)=45 minutes (tourner le patient, } \\
\text { aide en matière de mobilité, autres soins } \\
\text { directs reliés à la chimiothérapie par voie IP) } \\
\text { - Voie intravésiculaire=30 minutes }\end{array}$ \\
\hline
\end{tabular}


retenu tous les protocoles actuellement utilisés auxquels on a ajouté par la suite les protocoles dont on anticipe une utilisation croissante, ce qui a donné un total de 177 protocoles examinés. Ce chiffre (177) reflétait le nombre total de protocoles particuliers.

\section{Éléments de la charge de travail}

Le tableau 2 dégage chaque élément de la charge de travail et la durée de temps associée. Ces éléments comprennent les suivants: accès veineux, branchement et débranchement d'intraveineuse; temps de préparation; enseignement aux patients, etc. La prochaine étape amenait le groupe d'experts à aborder et à étudier la durée de temps correspondant à chaque élément de la charge de travail et à convenir d'une valeur; par exemple, la documentation exige 15 minutes; cependant, le nombre de prémédications requises pour un protocole donné varie selon le nombre de médicaments compris: 2 ou moins exigent 5 minutes; 3 ou plus exigent davantage de temps. La quantité d'hydratation par intraveineuse requise pour une prestation sécuritaire des soins aux patients dépendait du protocole. Par exemple, l'hydratation requise pour le cisplatine s'élevait à 5 minutes tandis que d'autres médicaments ne nécessitent aucune hydratation préou post-chimiothérapie. Le temps associé à la réaction était un autre domaine exigeant à la fois les connaissances expertes des membres du groupe et leurs connaissances sur les types de médicaments. Par exemple, certains protocoles entraînent un risque élevé de réaction et exigent donc une évaluation et une prise en charge infirmières plus poussées. Lorsque le risque de réaction est élevé, la durée de temps attribuée au protocole était de 20 à 30 minutes; en cas de faible risque de réaction, la durée de temps infirmier était comprise entre 5 et 10 minutes. Le groupe a consacré une quantité de temps considérable à chacune des définitions et des exigences temporelles, en réexaminant les décisions basées sur chacun des protocoles.

\section{Intensité de la charge de travail}

La durée de temps attribuée à la charge de travail associée à un protocole comprenait de nombreux éléments tels que les suivants: accès vasculaire, branchement et débranchement de perfusion dans une veine périphérique ou centrale; temps de préparation-étude des ordonnances, des analyses sanguines; enseignement aux patients; documentation; évaluation de la toxicité et gestion des symptômes; et enfin, médicaments additionnels requis, etc. Le tableau 2 présente tous les éléments de la charge de travail et les temps afférents y compris les soins directs aux patients et les éléments indirects depuis l'accès aux veines jusqu'aux affectations de temps concernant les pratiques en matière de contrôle des infections (colonnes A à L). Des domaines particuliers ont reçu une variable selon le nombre de prémédications exigé (F); la durée des soins infirmiers requis pour l'administration de médicaments particuliers comme les anticorps monoclonaux $(\mathrm{G})$; le temps d'observation requis (I); et le risque de réactions (J). Il convient de remarquer qu'une recension de la littérature a également été effectuée pour ces dimensions particulières afin d'intégrer dans le calcul

\begin{tabular}{|c|c|c|c|}
\hline Colonne & Élément de la & \multicolumn{2}{|c|}{ Définitions de chaque élément de la charge de travail associée à un protocole } \\
\hline $\mathrm{H}:$ & - Hydratation & $\begin{array}{l}\text { - Pré- et post-hydratation avec le cisplatine }=5 \\
\text { minutes } \\
\text { - Infusions de } \mathrm{Ca} / \mathrm{Mg} \text { pré- et post- } \\
\text { oxaliplatine }=5 \text { minutes }\end{array}$ & - Autre hydratation=5 minutes \\
\hline I: & Observation posttraitement & $\begin{array}{l}\text { - Période d'observation d'1 heure =10 minutes } \\
\text { de charge de travail d'infirmière }\end{array}$ & $\begin{array}{l}\text { - Période d'observation de } 30 \text { minutes }=5 \\
\text { minutes de charge de travail d'infirmière }\end{array}$ \\
\hline $\begin{array}{l}\text { J.i. \& } \\
\text { J.ii.: }\end{array}$ & $\begin{array}{l}\text { Réaction moyenne } \\
\text { Probabilité élevée }=7,5 \\
\text { minutes } \\
\text { Probabilité moyenne }=2,5 \\
\text { minutes } \\
\begin{array}{l}\text { Probabilité faible }=0,75 \\
\text { minute }\end{array}\end{array}$ & $\begin{array}{l}\text { - Appeler le médecin } \\
\text { - Signes vitaux, interventions reliées aux signes } \\
\text { vitaux } \\
\text { - Administration des médicaments } \\
\text { - Intervention d'une deuxième infirmière } \\
\text { Réaction moyenne: } \\
\text { - Probabilité élevée: } 20-30 \text { minutes } \\
\text { de temps de soins inf. exigés pour la } \\
\text { réaction: asparaginase en IV, carboplatine, } \\
\text { oxaliplatine, paclitaxel, docetaxel, rituximab } \\
\text { - Probabilité moyenne: } 10-15 \text { minutes de } \\
\text { temps de soins inf. exigés pour la réaction: } \\
\left.\text { doxorubicine liposomale pégylée (Caelyx }{ }^{\circledR}\right) \text {, } \\
\text { interféron-alfa-2b, paclitaxel hebdomadaire, } \\
\text { étoposide, cetuximab, trastuzumab, } \\
\text { temsirolimus } \\
\text { - Faible probabilité: } 5 \text { to } 10 \text { minutes de } \\
\text { temps de soins inf. exigés pour la réaction: } \\
\text { bléomycine, asparaginase par voie IM }\end{array}$ & $\begin{array}{l}\text { - Probabilité d'une réaction: } \\
\text { - Élevée: } 30 \% \\
\text { - Moyenne: } 20 \% \\
\text { - Faible: } 10 \% \\
\text { - Temps de réaction moyen par cycle }= \\
\text { probabilité } \times \text { temps moyen de réaction } \\
\text { - Élevé: } 7,5 \text { minutes }=0,3 \times 25 \text { minutes } \\
\text { - Moyen: } 2,5 \text { minutes }=0,2 \times 12,5 \text { minutes } \\
\text { - Faible: } 0,75 \text { minute }=0,1 \times 7,5 \text { minutes }\end{array}$ \\
\hline K: & $\begin{array}{l}\text { Entrée et sortie du patient } \\
5 \text { minutes }\end{array}$ & $\begin{array}{l}\text { - Avertir/appeler le patient pour qu'il vienne } \\
\text { dans la salle } \\
\text { - Accompagner le patient jusqu'au fauteuil } \\
\text { assigné et lui fournir l'aide nécessaire }\end{array}$ & $\begin{array}{l}\text { - Vérification/identification du patient } \\
\text { - Éléments de confort du patient } \\
\text { - Se débarrasser de tout le matériel utilisé pour } \\
\text { la dispensation d'agents cytotoxiques }\end{array}$ \\
\hline \multirow[t]{2}{*}{ L: } & $\begin{array}{l}\text { Pratiques de contrôle des } \\
\text { infections }-5 \text { minutes }\end{array}$ & \multicolumn{2}{|c|}{$\begin{array}{l}\text { - Isolement } \\
\text { - Prendre les mesures pour que le personnel nettoie le fauteuil ou le lit }\end{array}$} \\
\hline & Temps fixe total & \multicolumn{2}{|l|}{ Colonnes $\mathrm{A}+\mathrm{B}+\mathrm{C}+\mathrm{D}+\mathrm{E}+\mathrm{F}+\mathrm{K}+\mathrm{L}$} \\
\hline \multirow[t]{2}{*}{ M: } & $\begin{array}{l}\text { Ajustement multitâche de } \\
33 \% \text { du total fixe }\end{array}$ & \multicolumn{2}{|l|}{$(\text { Colonnes } \mathrm{A}+\mathrm{B}+\mathrm{C}+\mathrm{D}+\mathrm{E}+\mathrm{F}+\mathrm{K}+\mathrm{L})^{*} 1 / 3$} \\
\hline & $\begin{array}{l}\text { Temps lié à la charge de } \\
\text { travail infirmière }\end{array}$ & \multicolumn{2}{|l|}{ Colonnes $\mathrm{G}+\mathrm{H}+\mathrm{I}+\mathrm{J}+\mathrm{M}$} \\
\hline
\end{tabular}


des temps les meilleures données probantes disponibles. Par exemple, la variation de la probabilité d'une réaction (élevée, moyenne, faible) a été déterminée pour des médicaments particuliers à partir des données probantes émanant de la littérature et de la pratique clinique. Le groupe d'experts a également cerné la nécessité d'attribuer un supplément de temps à l'administration de protocoles par la voie intrapéritonéale ou par la voie intravésiculaire. Étant donné que ses travaux impliquaient la mesure du temps lié aux soins infirmiers, le groupe a reconnu qu'il lui fallait tenir compte du fonctionnement multitâche des infirmières. Afin d'incorporer la notion de fonctionnement multitâche, on a d'abord calculé le temps total fixe en ajoutant les facteurs temps de l'ensemble des colonnes A, B, C, D, E, F, K et L.

\section{Le processus d'examen}

Un examen détaillé des processus liés à la charge de travail et la comparaison entre les durées associées aux tâches considérées individuellement et celles associées au fonctionnement multitâche nous ont permis de cerner $33 \%$ à titre de facteur additionnel pertinent. Par conséquent, les durées fixes ont été multipliées par $33 \%$ afin de prendre en compte la participation des infirmières à des tâches multiples (facteurs communs) et auprès de patients multiples lorsqu'elles œuvrent dans les salles de chimiothérapie. Les calculs finals en vue de déterminer les valeurs temporelles de l'intensité de la charge de travail en soins infirmiers ont été réalisés en ajoutant les facteurs temps des colonnes $\mathrm{G}, \mathrm{H}$, I, J et M, et en calculant l'ajustement relatif au fonctionnement multitâche. Ces facteurs $(G, H$, I, J) représentent la variation au sein d'un même protocole comme la réaction chez le patient, la durée de l'administration, l'hydratation et l'observation. Quoique tous ces calculs puissent paraître d'une certain lourdeur, l'utilisation de cette méthode qui consiste à ajouter les facteurs temps de chacun de ces éléments et l'ajustement relatif au fonctionnement multitâche a permis de déterminer la durée finale correspondant à la charge de travail infirmier pour chaque protocole. Les infirmières n'ont pas besoin de faire de calculs; le facteur temps correspondant à chaque protocole a été dégagé et est présenté dans le formulary (formulaire des médicaments) d'ACO.

Le tableau 3 présente huit protocoles afin d'illustrer la variation du facteur temps d'un protocole à l'autre. Par exemple, la charge de travail infirmière liée à un protocole de chimiothérapie administré par la voie intrapéritonéale exige davantage de temps d'observation, s'accompagne d'un risque plus élevé de réaction (PACLICISP-IP) et est supérieure à la charge associée à l'administration du protocole GEM-CISP.

\section{Discussion}

Ce modèle d'affectation des ressources a été élaboré en combinant: a) la demande de services selon les clientèles au niveau régional et ce, en fonction de l'incidence et de l'utilisation; et, b) la méthodologie d'établissement des coûts/du financement rajustée

\begin{tabular}{|c|c|c|c|c|c|c|c|c|c|c|c|c|c|c|c|c|c|}
\hline $\begin{array}{l}\text { Code de } \\
\text { protocole }\end{array}$ & Description du protocole & A & B & C & D & E & F & G & $\mathbf{H}$ & I & J.i. & J.ii. & $\mathbf{K}$ & $\mathbf{L}$ & \begin{tabular}{|l|} 
Temps \\
fixe \\
total
\end{tabular} & M & $\begin{array}{l}\text { Intensité de } \\
\text { la charge } \\
\text { de travail } \\
\text { infirmière }\end{array}$ \\
\hline DOCETAX-100 & $\begin{array}{l}\text { SEIN, GASTRO-INTESTINAL, } \\
\text { GÉNITO-URINAIRE-DOCETAXEL } \\
\text { Q21 JOURS }\end{array}$ & 20 & 15 & 15 & 15 & 15 & 5 & 15 & 0 & 0 & Élevé & 7,5 & 5 & 5 & 95 & 31,7 & 54 \\
\hline $\begin{array}{l}\text { FOLFIRI- } \\
\text { BEVACIZUMAB }\end{array}$ & $\begin{array}{l}\text { GASTRO-INTESTINAL- } \\
\text { COLORECTAL, IRINOTÉCAN, } \\
\text { FLUOROURACIL IV en perfusion } \\
\text { continue, LEUCOVORIN, } \\
\text { BEVACIZUMAB }\end{array}$ & 20 & 15 & 15 & 15 & 15 & 5 & 35 & 0 & 0 & 0 & 0 & 5 & 5 & 95 & 31,7 & 67 \\
\hline GEM-CISP & $\begin{array}{l}\text { GÉNITO-URINAIRE-VESSIE OU } \\
\text { POUMON OU TÊTE ET COU OU } \\
\text { SEIN OU HÉMATOLOGIE OU } \\
\text { GASTRO-INTESTINAL-BILIAIRE, } \\
\text { GEMCITABINE, CISPLATINE }\end{array}$ & 20 & 15 & 15 & 15 & 15 & 5 & 10 & 5 & 0 & 0 & 0 & 5 & 5 & 95 & 31,7 & 47 \\
\hline CHOP & $\begin{array}{l}\text { HÉMATOLOGIE - LYMPHOME } \\
\text { NON HODGKINIEN- } \\
\text { CYCLOPHOSPHAMIDE, } \\
\text { DOXORUBICINE, VINCRISTINE, } \\
\text { PREDNISONE }\end{array}$ & 20 & 15 & 15 & 15 & 15 & 5 & 35 & 0 & 0 & 0 & 0 & 5 & 5 & 95 & 31,7 & 67 \\
\hline $\begin{array}{l}\text { FLUD-CYCLO- } \\
\text { RITUXIMAB }\end{array}$ & $\begin{array}{l}\text { HÉMATOLOGIE-FLUDARABINE, } \\
\text { CYCLOPHOSPHAMIDE, } \\
\text { RITUXIMAB (aussi appelé } \\
\text { «FCR ») }\end{array}$ & 20 & 15 & 15 & 15 & 15 & 5 & 20 & 0 & 5 & Élevé & 7,5 & 5 & 5 & 95 & 31,7 & 64 \\
\hline PACLICISP-IP & $\begin{array}{l}\text { GYNÉCOLOGIQUE-PACLITAXEL, } \\
\text { IP CISPLATINE }\end{array}$ & 40 & 15 & 15 & 15 & 15 & 7 & 45 & 5 & 10 & Élevé & 7,5 & 5 & 5 & 117 & 39,0 & 107 \\
\hline BCG & $\begin{array}{l}\text { GÉNITO-URINAIRE-BCG } \\
\text { (INTRAVÉSICULAIRE) }\end{array}$ & 0 & 15 & 15 & 15 & 15 & 5 & 30 & 0 & 0 & 0 & 0 & 5 & 5 & 75 & 25,0 & 55 \\
\hline $\begin{array}{l}\text { AC-TAXOL- } \\
\text { TRASTUZ }\end{array}$ & $\begin{array}{l}\text { SEIN-DOXORUBICINE, } \\
\text { CYCLOPHOSPHAMIDE (AC), PUIS } \\
\text { PACLITAXEL, TRASTUZUMAB }\end{array}$ & 20 & 15 & 15 & 15 & 15 & 7 & 25 & 0 & 5 & Élevé & 7,5 & 5 & 5 & 97 & 32,3 & 70 \\
\hline
\end{tabular}


en fonction des patients et des prestataires. Le système de financement était d'une capacité limitée pour ce qui est de refléter la complexité de la thérapie générale et des soins aux patients associés aux protocoles, d'évoluer au fur et à mesure de l'apparition de nouveaux protocoles et enfin, de soutenir la durabilité des cliniques d'oncologie communautaires. Une étude récemment publiée menée dans six centres de chimiothérapie de l'état de la Nouvelle-Galles du Sud, en Australie, a défini, à titre de composantes essentielles de la détermination de la charge de travail infirmière, les facteurs de l'enseignement aux patients, de l'évaluation du patient, de l'administration et de la communication avec le patient (De Raad et al., 2010). Dans leurs travaux, ces auteurs reconnaissent et confirment que les soins directs dépassent largement l'administration des médicaments.

\section{Implications pour le système}

Les ressources infirmières assignées à un cas individuel varient selon le diagnostic et le plan de traitement. Un établissement qui traite des protocoles exigeant davantage de ressources aura un indice des groupes clients supérieur à la moyenne provinciale (voir la figure 1). De même, les établissements peuvent décider d'utiliser un protocole différent pour un diagnostic particulier qui peut s'accompagner d'un nombre de visites anticipées qui diffère de celui des visites réelles (voir la figure 2). On dénombre, dans la base de données d'ACO intitulée Activity Level Reporting (laquelle est une composante clé de l'Ontario Cancer Registry), 177 protocoles distincts qui sont dispensés

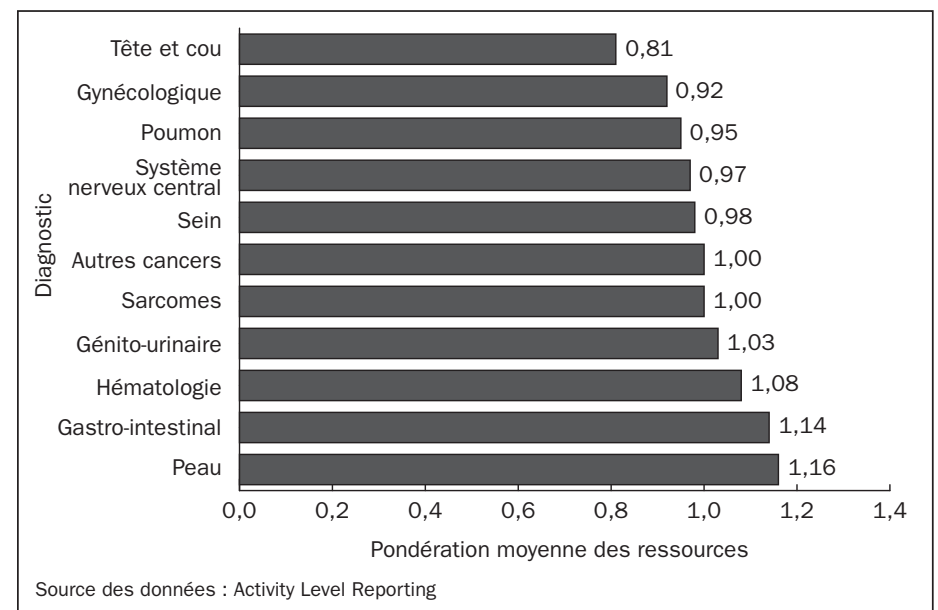

Figure 1. Pondération moyenne des ressources par diagnostic, 2009-10

\begin{tabular}{|c|c|c|c|c|c|c|}
\hline \multicolumn{3}{|c|}{ Établissement A } & \multirow{2}{*}{$\begin{array}{r}\text { Visites } \\
\text { réelles } \\
15\end{array}$} & \multirow{2}{*}{\begin{tabular}{r}
\multicolumn{1}{c}{$\begin{array}{c}\text { a } \\
\text { Visites } \\
\text { anticipées }\end{array}$} \\
10
\end{tabular}} & \multirow{2}{*}{$\begin{array}{c}\begin{array}{c}\text { b } \\
\text { Pondération } \\
\text { des ressources } \\
\text { par visite }\end{array} \\
0,13\end{array}$} & \multirow{2}{*}{$\begin{array}{c}\mathbf{a} \times \mathbf{b}=\mathbf{c} \\
\begin{array}{c}\text { Pondération } \\
\text { des ressources } \\
\text { totale }\end{array} \\
\mathbf{1 , 3}\end{array}$} \\
\hline Patient 1 & Protocole 5 & Diagnostic A & & & & \\
\hline Patient 2 & Protocole 1 & Diagnostic B & 2 & 3 & 0,3 & 0,9 \\
\hline $\begin{array}{l}\text { Indice de groupes clients de } \\
\text { l'établissement }\end{array}$ & & & \multicolumn{4}{|c|}{$(\mathbf{1}, \mathbf{3}+\mathbf{0 , 9}) \div 2=1,10$} \\
\hline Taux de financement par cas & & & \multicolumn{4}{|c|}{$3300 \$ \times 1,1=3630 \$$} \\
\hline
\end{tabular}

\begin{tabular}{|c|c|c|c|c|c|c|}
\hline \multicolumn{3}{|c|}{ Établissement B } & \multirow{2}{*}{$\begin{array}{r}\text { Visites } \\
\text { réelles } \\
17\end{array}$} & \multirow{2}{*}{\begin{tabular}{r}
\multicolumn{1}{c}{$\begin{array}{c}\text { a } \\
\text { Visites } \\
\text { anticipées }\end{array}$} \\
20
\end{tabular}} & \multirow{2}{*}{$\begin{array}{c}\text { b } \\
\begin{array}{c}\text { Pondération } \\
\text { des ressources } \\
\text { par visite }\end{array} \\
0,11\end{array}$} & \multirow{2}{*}{$\begin{array}{c}\mathbf{a} \times \mathbf{b}=\mathbf{c} \\
\begin{array}{c}\text { Pondération } \\
\text { des ressources } \\
\text { totale }\end{array} \\
\mathbf{2 , 2}\end{array}$} \\
\hline Patient 1 & Protocole 10 & Diagnostic A & & & & \\
\hline Patient 2 & Protocole 1 & Diagnostic B & 2 & 3 & 0,3 & 0,9 \\
\hline $\begin{array}{l}\text { Indice de groupes clients de } \\
\text { l'établissement }\end{array}$ & & & \multicolumn{4}{|c|}{$(\mathbf{2}, \mathbf{2}+\mathbf{0 , 9}) \div 2=1,55$} \\
\hline Taux de financement par cas & & & \multicolumn{4}{|c|}{$3300 \$ \times 1,55=5115 \$$} \\
\hline
\end{tabular}

Figure 2. dans les programmes de cancérologie de la province. Les protocoles sont reliés aux sièges du cancer et les variations concernent le dosage et l'utilisation d'un protocole en fonction d'un siège particulier. Cela signifie que certains protocoles nécessitent plus ou moins de visites selon des facteurs propres au patient. Le nombre de visites et le nombre de cas par siège de cancer ont été examinés dans le cadre de la détermination globale du groupe clients.

Les travaux du groupe d'experts ont orienté la distribution de l'ensemble des fonds assignés à la thérapie générale de manière à mieux refléter la teneur en ressources. Ce projet visait à développer un modèle de financement équitable tenant compte des protocoles réellement dispensés et établissant une correspondance entre le financement et les objectifs en matière de qualité. L'affectation du financement est actuellement basée sur le nombre de nouvelles consultations effectuées par les oncologues médicaux, ce qui donne un montant de financement de base. De plus, un financement annuel supplémentaire est nécessaire pour prendre en charge la croissance du nombre de nouveaux cas ajoutés à la charge de travail. La pondération de la consommation des ressources constitue une méthodologie qui permet de coordonner le financement et la planification de la prestation de la thérapie générale et de promouvoir un emploi efficace des ressources au niveau local. Les données sur les protocoles incluant l'utilisation de la thérapie générale font l'objet d'une divulgation régulière dans les programmes de cancérologie et peuvent servir à adapter le financement supplémentaire aux ressources réelles requises pour la dispensation des soins. ACO a posé l'hypothèse d'un coût moyen de 1,0 et a déterminé tous les protocoles en relation avec ce coût. Cela signifie qu'un protocole d'une pondération de 1,2 coûte $20 \%$ de plus que le coût provincial moyen. Tous les protocoles dispensés par un programme donné servent au calcul de l'indice d'un groupe clients (IGC) particulier. Afin de valider plus à fond l'IGC, le groupe d'experts a examiné les résultats sur une période de trois ans et a constaté que l'IGC était stable pour l'ensemble des programmes de cancérologie. ACO s'est engagé à actualiser annuellement chaque IGC des programmes en fonction des protocoles réellement dispensés.

L'outil de pondération de la consommation des ressources a permis au système ontarien de lutte contre le cancer d'élaborer une plateforme objective à l'aide de laquelle il peut négocier, avec le Ministère de la Santé et des Soins de longue durée, un financement reflétant l'état actuel de la thérapie générale. L'outil et la méthodologie ont été passés en revue par tous les programmes de cancérologie de l'Ontario. En outre, plusieurs de ces programmes se sont servis de l'information pour élaborer des outils d'ordonnancement des soins infirmiers dans les salles de chimiothérapie ambulatoire.

\section{Implications pour les soins infirmiers}

Ces travaux ont porté fruit en approfondissant les connaissances sur les enjeux liés à la charge de travail des infirmières et la compréhension de ces derniers. La durée finale attribuée aux tâches infirmières (consulter le tableau 2) montre bien que les protocoles nécessitent des quantités de temps variées pour la prestation des soins aux patients; ceci a confirmé l'intuition des gestionnaires qu'il leur fallait rajuster la quantité totale de soins infirmiers disponibles lorsque des protocoles étaient modifiés ou lorsque de nouveaux protocoles étaient mis en œuvre. Il convient de remarquer que la réussite de ce projet est due à la volonté du bailleur de fonds et des intervenants d'élaborer une formule de financement équitable et pertinente du point de vue clinique qui faciliterait l'intégration continue de la planification, des normes et du financement. 
Puisque le modèle devait servir à justifier le financement, il a fait l'objet d'un examen extrêmement rigoureux de la part des utilisateurs, lequel a abouti à un système fondé sur la composition de la clientèle à la fois robuste et complet.

L'examen individuel des protocoles a également débouché sur des discussions importantes sur la variation des pratiques entre les programmes. Cette variation sur le plan de l'utilisation des protocoles auprès de clientèles similaires révélait que des améliorations de l'efficacité étaient généralement possibles au sein du système en substituant des protocoles exigeant de moindres ressources à ceux qui en exigent davantage. En outre, la grande diversité des protocoles employés pour chaque siège de cancer indiquait qu'il était possible d'accroître la normalisation à l'échelle de la province.

Les travaux réalisés en vue de définir la pondération de la consommation des ressources pour les protocoles complexes permettent aux infirmières et aux gestionnaires de cerner les protocoles et exigences de soins associés à une charge de travail élevée et leur donnent l'opportunité de planifier les niveaux de dotation en personnel en vue de répondre aux besoins des patients atteints de cancer. Ils ont débouché sur la création d'un modèle de financement synchronisé pour les soins infirmiers associés aux modalités de thérapie générale. L'utilisation éventuelle d'un modèle de financement dynamique est essentielle dans un contexte de contraintes financières si l'on veut financer les soins nécessaires et optimiser les améliorations en matière de qualité.

\section{Implications pour les patients}

La pondération de la consommation des ressources a des conséquences indirectes pour les patients puisque, dorénavant, les affectations des ressources infirmières se basent sur une méthodologie. On emploie désormais une approche plus uniforme de prestation des soins infirmiers incorporant les éléments directs et indirects. Les gestionnaires peuvent ainsi affecter les infirmières de manière à répondre aux besoins complexes des patients recevant tous les protocoles de chimiothérapie.

\section{RÉFÉRENCES}

Chabot, G., \& Fox, M. (2005). The creation of a patient-classification system in an outpatient infusion center setting. Oncology Nursing Forum, 32(3), 535-538.

Colombo, A., Solberg, B., Vanderhoeft, E., Ramsay, G., \& Schouten, H. (2005). Measurement of nursing care time of specific interventions on a hematology-oncology unit related to diagnostic categories. Cancer Nursing, 28(6), 476-480.

Craig, K., \& Huber, D. (2007). Acuity and case management: A healthy dose of outcomes (Part II). Professional Case Management, 12(4), 199-210.

Cusack, G., Jones-Wells, A., \& Chisholm, L. (2004a). Patient intensity in an ambulatory oncology research center: A step forward for the field of ambulatory care (Part I). Nursing Economics, 22(2), 58-63.

Cusack, G., Jones, A., \& Chisholm, L. (2004b). Patient intensity in an ambulatory oncology research center: A step forward for the field of ambulatory care (Part III). Nursing Economics, 22(4), 193-195.

De Raad, J., van Gool, K., Haas, M., Haywood, P., Faedo, M., Gallego, G., Pearson, S., \& Ward, R. (2010) Nursing takes time: Workload associated with administering cancer protocols. Clinical Journal of Oncology Nursing, 14(6), 735-741.

Harper, K., \& McCully, C. (2007). Acuity systems dialogue and patient classification system essentials. Nursing Administration Quarterly, 31(4), 284-299.

Huber, D., \& Craig, K. (2007a). Acuity and case management: A healthy dose of outcomes (Part I). Professional Case Management, 12(3), 132-44.

Huber, D., \& Craig, K. (2007b). Acuity and case management: A healthy dose of outcomes (Part III). Professional Case Management, 12(5), 254-69.

\section{Limitations de l'outil}

L'outil a été examiné par divers groupes de l'Ontario (Oncology Nursing Provincial Committee, Regional Systemic Treatment Program, Provincial Leadership Council). Cependant, un examen global n'a pas été effectué en dehors de l'Ontario. La capacité de l'outil à s'adapter généralement aux programmes de cancérologie externes au contexte ontarien devra être déterminée par d'autres autorités de santé à la lumière de son application à des protocoles particuliers.

\section{Conclusion}

Dans les milieux de prestation de soins de cancérologie complexes, il est essentiel que les besoins en ressources infirmières soient reliés aux besoins des patients recevant soins et traitements. Les travaux de ce groupe d'experts avaient pour but de développer une méthodologie de pondération de la consommation des ressources axée sur les protocoles qui inclurait l'ensemble des éléments de soins, à la fois directs et indirects. La méthode permet aux infirmières et aux gestionnaires de dégager les protocoles à forte consommation de ressources ainsi que les exigences de soins infirmiers qui appuient les changements en matière de dotation et fournit les données justifiant les coûts associés aux diverses clientèles.

\section{Remerciements}

Les auteurs aimeraient reconnaître avec gratitude les contributions que les personnes suivantes ont apportées à cet article : Linda RobbBlenderman (Hôpital général de Kingston), Tracy McQueen (Hôpital général de Kingston), Mychele Sabourin (Programme régional de cancérologie de l'Hôpital d'Ottawa), Leila Shenouda (Hôpital Princess Margaret), Jayesh Patel (Hôpital Princess Margaret), Jonathan Lam (ACO), Julia Gao (ACO) et Naomi Greenberg (ACO).

Jones, A., Cusack, G., \& Chisholm, L. (2004). Patient intensity in an ambulatory oncology research center: A step forward for the field of ambulatory care (Part II). Nursing Economics, 22(3), 120-123.

Langhorn, M., \& Morrison, C. (2001a). Redesigning processes in ambulatory chemotherapy: Creating a patient appointment scheduling system (Part I). Canadian Oncology Nursing Journal, 11(2), 109-110.

Langhorn, M., \& Morrison, C. (2001b). Redesigning processes in ambulatory chemotherapy: Creating a patient appointment scheduling system (Part II). Canadian Oncology Nursing Journal, 11(3), 157-159.

Moore, M., \& Hastings, C. (2006). The evolution of an ambulatory nursing intensity system-Measuring nursing workload in a day hospital setting. Journal of Nursing Administration, 36(5), 241-248.

Morris, R., MacNeela, P., Scott, A., Treacy, P., \& Hyde, A. (2007). Reconsidering the conceptualization of nursing workload: Literature review. Journal of Advanced Nursing, 57(5), 463-471.

The Systemic Therapy Advisory Working Group [STAWG] (2002). Report of the systemic therapy advisory working group, Ontario (unpublished report). Presented to the Ministry of Health and Long Term Care and Cancer Care Ontario.

The Systemic Therapy Task Force. (2000). Systemic therapy task force report. Ontario: Presented to Cancer Care Ontario (unpublished report). April 2000.

Unruh, L. (2008). Nurse staffing and patient nurse and financial outcomes. The American Journal of Nursing, 108(1), 62-71.

Welton, J.M., Zone-Smith, L., \& Bandyopadhyay, D. (2009). Estimating nursing intensity and direct cost using the nurse-patient assignment. Journal of Nursing Administration, 39(6), 276-284. 